\title{
A Truthful Optimal Path Auction for DSA Networks
}

\author{
Swastik Brahma and Mainak Chatterjee \\ Electrical Engineering and Computer Science \\ University of Central Florida \\ Orlando, FL 32816 \\ Email: \{sbrahma, mainak\}@eecs.ucf.edu
}

\begin{abstract}
In this paper, we address the problem of incentive based routing in DSA networks, where each secondary node having a certain capacity incurs a cost for routing traffic through it. We propose a path auction scheme in which each secondary node announces its cost and capacity to the routing mechanism, both of which are considered as private information known only to the node. We design a route selection mechanism and a pricing function that can induce nodes to reveal their cost and capacity honestly, while minimizing the payment that needs to be given to the nodes. Earlier works either do not consider the capacity constraint of the nodes (VCG based least cost path routing [4], [6], [10]), or consider the capacity constraint without enforcing truthful capacity reporting [8], or require special hardware/software to be installed in the nodes for truthful revelation of a node's private information [11]. Moreover, the VCG based mechanisms focus on finding a least cost path, which may not be the path that requires the least payment. For deploying our path auction based routing mechanism in DSA networks, we provide a polynomial time algorithm to find the optimal route over which traffic should be routed and to compute the payment that each node should receive. The performance of our scheme is evaluated via extensive simulations.
\end{abstract}

\section{INTRODUCTION}

The limitations of static spectrum allocation have motivated a paradigm shift from static spectrum allocation towards a more 'liberalized' notion of spectrum management in which secondary users can borrow idle spectrum from primary spectrum licensees, without causing harmful interference to the latter- a notion commonly referred to as dynamic spectrum access (DSA) or open spectrum access [1]. However, to motivate primary users to allow usage of their idle spectrum for secondary use, an incentive, generally in the form of monetary benefit, has to be given to them. Such economic considerations have been reflected in recent research which mostly propose auctions as the means of trading spectrum between primary and secondary entities [7], [13], [14].

Since secondary users acquire licenses from the primaries by paying for the same, incentives have to be given to the secondary users to provide services, such as routing, to outside entities. To forward traffic, each secondary node would incur a cost because of using the spectrum it has acquired by paying a price. Moreover, using the acquired spectrum, each node can sustain a certain capacity. Clearly, secondary nodes will not have an incentive to relay traffic unless they are compensated for the costs they incur in forwarding traffic. Also, for a routing mechanism to be stable, the amount of traffic that a node forwards should not exceed its capacity. For a flow from a

This research was sponsored by the National Science Foundation, under source $s$ to a destination $d$ in an DSA network, the routing problem (from an economic perspective) is to find a path (or a set of paths) from $s$ to $d$, that has sufficient capacity to route the given flow, and using which the total payment that needs to be made to relaying nodes is minimized.

The complexity in solving the routing problem lies in the fact that a node can potentially profit by dishonestly reporting its cost and/or capacity to the routing mechanism, which is not aware of the actual cost and capacity of a node. Thus, a desirable feature is that the routing mechanism should be able to induce nodes to truthfully reveal their cost and capacity by giving them incentives for doing so. Moreover, the mechanism should be such that the total payment that needs to be made to the nodes while giving them such incentives is minimized.

Most past work have focused on truthful revelation of the cost perspective alone and does not consider capacity constraint [4], [6], [10]. These works consider finding a least cost path (LCP) from $s$ to $d$ with each agent (a node or an edge) having a per packet cost for relaying traffic. To induce the agents to reveal their costs truthfully, the pricing scheme in these works is based on the Vickrey-Clarke-Groves (VCG) class of mechanisms [3], [5], [12]. In this scheme, the payment $p^{e}$ given to an agent $e$ is 0 if $e$ is not in the LCP and $p^{e}=d_{G \mid e=\infty}-d_{G \mid e=0}$ if it is. Here, $d_{G \mid e=\infty}$ is the cost of the least cost path that does not contain $e$, and $d_{G \mid e=0}$ is the cost of the least cost path when the cost of $e$ is assumed to be zero. It is worth emphasizing here that LCP need not necessarily be the path that minimizes the total payment that needs to be made to the agents that help forward traffic. The authors in [8] consider the capacity constraint but do not enforce the nodes to report their capacity truthfully, while the work in [11] requires tamper-proof hardware/software to be installed in the nodes to enforce truthful capacity reporting.

In contrast to the previous works, our model theoretically ensures that no node has any incentive to dishonestly declare its cost or capacity. Based on the optimal auction theory by Myerson [9], we adopt the approach of Bayesian based mechanism design to ensure that honestly revealing its cost and capacity forms a dominant strategy for every node in the path auction game. The key points of our work are as follows.

- We propose a path auction based routing mechanism in which nodes announce their cost and capacity, based on which a multi-path route is chosen and payments are made to the nodes. The route selection mechanism and the payment function ensures that all nodes can maximize their profit by truthfully reporting their cost and capacity, while the payment to be made to the nodes is minimized. 
- We provide a polynomial time algorithm to implement the routing mechanism in DSA networks.

- We model cost of a node and its capacity as random variables. This serves a two-fold purpose- $a$ ) it helps tame the uncertainty regarding the actual cost and capacity of a node; and, $b$ ) it helps reflect the heterogenous nature of the nodes in a DSA network in terms of both space and time variation of the cost and capacity of nodes.

\section{Network Model and Problem Formulation}

\section{A. Network Model and Assumptions}

We consider a DSA network whose topology is depicted by the graph $G=(V, E)$. Here $V$ is the set of secondary nodes and $E$ is the set of links between the secondary nodes. Each node $j \in V$ acquires (leases) a certain amount of spectrum which can sustain a capacity $c_{j}$, measured in packets per unit time. In return, node $j$ pays a cost $m_{j}$ per unit time, for the time it uses the spectrum, to the primary owner. Cost $m_{j}$ as well as the capacity, $c_{j}$, are considered to be private information only known to node $j$. Thus, the type of node $j \in V$ is defined by the 2-tuple $t_{j}=\left\{m_{j}, c_{j}\right\}$. It is worth emphasizing that, even though we consider a packet to be the smallest granularity of data transfer, our analysis would also hold true even if we consider other granularities such as bits.

Consider a traffic flow that originates from source node $s$ and terminates at destination node $d$. Let $N$ represent the set of candidate secondary nodes available for routing the flow. Thus, $N=V \backslash\{s, d\}$. We label the nodes in $N$ from 1 to $n$, i.e., $N=\{1 \cdots n\}$. Before the flow begins, each node $j \in N$ reports its type to the routing mechanism as the 2tuple $\hat{t}_{j}=\left\{\hat{m}_{j}, \hat{c}_{j}\right\}$. A truthful mechanism has to guarantee that no node $j \in N$ has an incentive to lie about its type, i.e., the reported type $\hat{t}_{j}=t_{j}, \forall j \in N\left(\hat{m}_{j}=m_{j}\right.$ and $\left.\hat{c}_{j}=c_{j}\right)$.

The routing mechanism, however, is not aware of the true type of an intermediate node $j \in N$. To this end, we will tackle the problem using the conventional economics approach of Bayesian mechanism design, modeling $m_{j}$ and $c_{j}$ as random variables. We assume that the uncertainty about the type of node $j \in N$ can be described by two continuous probability density functions (p.d.f)- one for the cost, $m_{j}$ and another for the capacity, $c_{j}$ - and that the functions are known to the mechanism. We denote the domain of the p.d.f $f_{j}^{M}\left(m_{j}\right)$ as $\left[a_{j}, b_{j}\right]$, where $a_{j} \leq b_{j}$ and $a_{j} \geq 0, b_{j} \geq 0$. Also, we denote the domain of the p.d.f $f_{j}^{C}\left(c_{j}\right)$ as $\left[v_{j}, w_{j}\right]$, where $v_{j} \leq w_{j}$ and $v_{j} \geq 0, w_{j} \geq 0$.

Cost, $m_{j}$, as well as the capacity, $c_{j}$, depend on the supply demand dynamics of spectrum availability in the vicinity of node $j$. Thus, we consider $m_{j}$ and $c_{j}$ to be dependent random variables. Let $f_{j}^{M C}$ be their continuous joint probability density function defined over the domain, $T_{j}$. Thus,

$$
T_{j}=\left[a_{j}, b_{j}\right] \times\left[v_{j}, w_{j}\right]
$$

Let $T$ denote the set of all possible combinations of types of the nodes in $N$. Thus,

$$
T=T_{1} \times \cdots \times T_{n}
$$

Now, if $t_{j}=\left\{m_{j}, c_{j}\right\}$ is the type of $j$, then the price that $j$ pays to the primary spectrum owner for transmitting a packet over its spectrum is $m_{j} / c_{j}$. We denote this price associated with the type of $j$ as $t_{j}^{p}$. Let $f_{j}^{P}$ be the probability density function of the continuous random variable $t_{j}^{p}$ over the domain,

$$
T_{j}^{p}=\left[\frac{a_{j}}{w_{j}}, \frac{b_{j}}{v_{j}}\right]
$$

Let $T^{p}$ denote the set of all possible combinations of the per packet prices that can be associated with the types of the nodes in $N$. Thus,

$$
T^{p}=T_{1}^{p} \times \cdots \times T_{n}^{p}
$$

Also, for node $j \in N$, let $T_{-j}^{p}$ denote the set of all possible combinations of the per packet prices that can be associated with the types of the nodes in $N$ other than $j$, so that

$$
T_{-j}^{p}=\underset{\substack{i \in N \\ i \neq j}}{ } T_{i}^{p}
$$

We assume that the types of the nodes in $N$ are stochastically independent from each other. Thus, the joint density function on $T^{p}$ for the vector $t^{p}=\left(t_{1}^{p}, \cdots, t_{n}^{p}\right)$ of individual per packet prices associated with the vector $t=\left(t_{1}, \cdots, t_{n}\right)$ of individual types is,

$$
f^{P}\left(t^{p}\right)=\prod_{j \in N} f_{j}^{P}\left(t_{j}^{p}\right)=\prod_{j \in N} f_{j}^{P}\left(m_{j} / c_{j}\right)
$$

Also, we assume that a node $j \in N$ assesses the uncertainty about the types of the other nodes in $N$ in the same way as the routing mechanism does. Consequently, both the mechanism and node $j$ assess the joint density function on $T_{-j}^{p}$ for the vector $t_{-j}^{p}=\left(t_{1}^{p}, \cdots, t_{j-1}^{p}, t_{j+1}^{p}, \cdots, t_{n}^{p}\right)$ associated with the vector $t_{-j}=\left(t_{1}, \cdots, t_{j-1}, t_{j+1}, \cdots, t_{n}\right)$ of individual types of all nodes in $N$ except $j$ as,

$$
f_{-j}^{P}\left(t_{-j}^{p}\right)=\prod_{\substack{i \in N \\ i \neq j}} f_{i}^{P}\left(t_{i}^{p}\right)
$$

\section{B. Problem Formulation}

The optimal routing mechanism design problem is to devise a path auction mechanism such that the expected payment made by the end-user to the nodes in $N$ to have its traffic routed from $s$ to $d$ is minimized, while enforcing nodes to reveal their type to the mechanism truthfully. The end-user can correspond to either $s$ or $d$. In this paper we consider the direct revelation mechanism class, where the nodes in $N$ simultaneously and confidentially announce their respective type $t_{j}=\left\{m_{j}, c_{j}\right\}, j \in N$, to the routing mechanism. The mechanism then determines on which path(s) to route the traffic from $s$ to $d$ and how much each node in $N$ should be paid, as some functions of the vector of per packet prices $t^{p}=\left(t_{1}^{p}, \cdots, t_{n}^{p}\right)$ associated with the vector of announced types $t=\left(t_{1}, \cdots, t_{n}\right)$.

Let $P^{s \rightarrow d}$ denote the set of all paths from $s$ to $d$, with each path consisting of nodes defined in $G$, and let $K=$ $\left|P^{s \rightarrow d}\right|$ with the paths in $P^{s \rightarrow d}$ labeled from 1 to $K$. The routing mechanism can then be described by a pair of outcome functions $(p, q)$ such that, $p_{j}\left(t^{p}\right)(j \in[1, n])$ is the amount of money paid to node $j$ for each packet delivered to $d$ by the nodes in $N$ (or, routes in $P^{s \rightarrow d}$ ) and $q_{i}\left(t^{p}\right),(i \in[1, K])$, is the fraction of traffic routed over path $i$.

We consider expected utility of the end-user in terms of the price that the end-user needs to pay for each packet from $s$ that is serviced (routed) by the underlying network and delivered to $d$. In section (III-B) we will discuss who actually makes 
the payments. The expected utility of the end-user from the routing mechanism described by $(p, q)$ is,

$$
U_{e}(p, q)=\int_{T^{p}}\left(\sum_{j \in N} p_{j}\left(t^{p}\right)\right) f^{P}\left(t^{p}\right) d t^{p} .
$$

where, $d t^{p}=d t_{1}^{p} \cdots d t_{n}^{p}$ and $d t_{j}^{p}=d\left(\frac{m_{j}}{c_{j}}\right), j \in[1, n]$. Similarly, we consider the expected utility of node $j \in N$ in terms of its profit per packet delivered to $d$ by the routes in $P^{s \rightarrow d}$. If the type of $j$ is $t_{j}=\left\{m_{j}, c_{j}\right\}$, then its expected utility from the auction mechanism is,

$U_{j}\left(p, q, t_{j}^{p}\right)=\int_{T_{-j}^{p}}\left(p_{j}\left(t^{p}\right)-t_{j}^{p} \sum_{i \in P^{s} \stackrel{j}{\rightarrow} d} q_{i}\left(t^{p}\right)\right) f_{-j}^{P}\left(t_{-j}^{p}\right) d t_{-j}^{p}$

where, $P^{s \stackrel{j}{\rightarrow} d}$ is the subset of paths in $P^{s \rightarrow d}$ that pass via node $j$. The objective of the path auction design problem is to shape the pair of outcome functions $(p, q)$, such that, the expected utility of the end-user as defined by equation (8) is minimized. However, there are certain constraints that must be imposed on $(p, q)$ to be able to describe a feasible auction mechanism. We describe these constraints below.

1. Individual Rationality Constraint: No node in $N$ can be forced to participate in the auction if its utility from routing traffic is negative. If a node does not participate in the auction, then clearly its utility would be zero. Thus, to ensure that all nodes in $N$ participate in the auction, the following individual rationality constraint must hold.

$$
U_{j}\left(p, q, t_{j}^{p}\right) \geq 0, \quad \forall j \in N, \forall t_{j}^{p} \in T_{j}^{p}
$$

2. Incentive Compatibility Constraint: To make the nodes in $N$ reveal their respective types truthfully, no node should be able to expect an extra profit from lying about its type. In other words, honestly revealing its own type should form a dominant strategy for every node in $N$ in the auction game. If node $j \in N$ reports its type as $\hat{t}_{j}=\left\{\hat{m}_{j}, \hat{c}_{j}\right\}$ while its true type is $t_{j}=\left\{m_{j}, c_{j}\right\}$, then its expected utility is given by,

$\int_{T_{-j}^{p}}\left(p_{j}\left(\hat{t}_{j}^{p}, t_{-j}^{p}\right)-t_{j}^{p} \sum_{i \in P^{s} \stackrel{j}{\rightarrow} d} q_{i}\left(\hat{t}_{j}^{p}, t_{-j}^{p}\right)\right) f_{-j}^{P}\left(t_{-j}^{p}\right) d t_{-j}^{p}$

where, $\left(\hat{t}_{j}^{p}, t_{-j}^{p}\right)=\left(t_{1}^{p}, \cdots, t_{j-1}^{p}, \hat{t}_{j}^{p}, t_{j+1}^{p}, \cdots, t_{n}^{p}\right)$ Thus, to ensure that no node in $N$ has an incentive to lie about its type, the following incentive compatibility condition must hold,

$$
\begin{aligned}
& U_{j}\left(p, q, t_{j}^{p}\right) \geq \\
& \int_{T_{-j}^{p}}\left(p_{j}\left(\hat{t}_{j}^{p}, t_{-j}^{p}\right)-t_{j}^{p} \sum_{\substack{i \in P^{s}{ }^{\prime} d \\
\forall j \in N}} q_{i}\left(\hat{t}_{j}^{p}, t_{-j}^{p}\right)\right) f_{-j}^{P}\left(t_{-j}^{p}\right) d t_{-j}^{p}, \\
& \forall j \in T_{j}^{p}, \forall \hat{t}_{j}^{p} \in T_{j}^{p}
\end{aligned}
$$

Notice that if $\hat{t}_{j}^{p}=t_{j}^{p}$, i.e., $\frac{\hat{c_{j}}}{\hat{c_{j}}}=\frac{m_{j}}{c_{j}}$, then the expected utility of $j$ by reporting type $\hat{t}_{j}$ is equal to $U_{j}\left(p, q, t_{j}^{p}\right)$. So node $j$ cannot expect to gain by reporting a type $\hat{t}_{j}$ if $\hat{t}_{j}^{p}=t_{j}^{p}$.

3. Traffic Allocation Constraint: Clearly, sum of the fraction of traffic allocated over all paths is 1 . Thus,

$\sum_{i=1}^{K} q_{i}\left(t^{p}\right)=1$ and $q_{i}\left(t^{p}\right) \geq 0, \quad \forall i \in[1, K], \forall t^{p} \in T^{p}$
4. Node Capacity Constraint: The total traffic rate that a node routes cannot exceed its capacity. Thus,

$$
R \sum_{i \in P^{s} \stackrel{j}{\rightarrow} d} q_{i}\left(t^{p}\right) \leq c_{j}, \quad \forall j \in N, \forall t^{p} \in T^{p}
$$

where, $R$ is the rate at which $s$ injects its traffic into the network.

\section{Path Auction Design}

Let $Q_{j}\left(q, t_{j}^{p}\right)$ denote the expected fraction of traffic that node $j \in N$ will be made responsible for forwarding due to a path auction mechanism $(p, q)$, given that $j$ 's type is $t_{j}=$ $\left\{m_{j}, c_{j}\right\}$. Thus, we can define,

$$
Q_{j}\left(q, t_{j}^{p}\right)=\int_{T_{-j}^{p}}\left(\sum_{i \in P^{s \stackrel{j}{\rightarrow} d}} q_{i}\left(t^{p}\right)\right) f_{-j}^{P}\left(t_{-j}^{p}\right) d t_{-j}^{p}
$$

Based on the above definition, we first present a simplified incentive compatibility constraint.

LEMMA 1: The incentive compatibility constraint presented in (12) holds if the following two conditions hold for $\forall j \in$ $N, \forall m_{j}, n_{j} \in\left[a_{j}, b_{j}\right], \forall c_{j}, d_{j} \in\left[v_{j}, w_{j}\right]$ :

1. if $m_{j} \leq n_{j}$ and $c_{j} \leq d_{j}$, then,

$$
\begin{gathered}
Q_{j}\left(q, \frac{n_{j}}{c_{j}}\right) \leq Q_{j}\left(q, \frac{m_{j}}{d_{j}}\right) \\
\text { 2. } U_{j}\left(p, q, \frac{m_{j}}{c_{j} / v_{j}}\right)=\int_{m_{j} / c_{j}}^{b_{j}} Q_{j}\left(q, \psi_{j}\right) d \psi_{j}+U_{j}\left(p, q, \frac{b_{j}}{v_{j}}\right)
\end{gathered}
$$

Proof: We omit the proof due to space constraints. The reader can refer to [2] for the proof.

Thus, $(p, q)$ can be said to describe an optimal path auction if and only if it minimizes $U_{e}(p, q)$ subject to the incentive compatibility constraints (16)-(17), individual rationality constraint (10), traffic allocation constraint (13), and node capacity constraint (14). Next, we simplify the optimization problem presented in section (II-B).

LEMMA 2: $(p, q)$ represents an optimal path auction mechanism if $q$ minimizes

$$
\int_{T^{p}}\left[\sum_{j \in N}\left(t_{j}^{p}+\frac{F_{j}^{P}\left(t_{j}^{p}\right)}{f_{j}^{P}\left(t_{j}^{p}\right)}\right) \sum_{i \in P^{s} \stackrel{j}{\rightarrow} d} q_{i}\left(t^{p}\right)\right] f^{P}\left(t^{p}\right) d t^{p}
$$

subject to constraints (13) and (14), and,

$$
\begin{aligned}
& p_{j}\left(t^{p}\right)=\frac{m_{j}}{c_{j}} \sum_{i \in P^{s} \stackrel{j}{\rightarrow} d} q_{i}\left(t^{p}\right)+\int_{m_{j} / c_{j}}^{b_{j} / v_{j}} \sum_{i \in P^{s} \stackrel{j}{\rightarrow} d} q_{i}\left(\psi_{j}, t_{-j}^{p}\right) d \psi_{j} \\
& \forall j \in N, \forall t^{p} \in T^{p}
\end{aligned}
$$

Proof: Please refer [2] for the proof.

Next, we will formulate lemma (2) in a deterministic manner.

LEMMA 3: For any given $t$ in $T$, the optimal traffic allocation over different routes in $P^{s \rightarrow d}$ is obtained if $q$ minimizes the following function for the corresponding $t^{p}$,

$$
\Phi\left(t^{p}\right)=\sum_{i=1}^{K}\left[\sum_{j \in P_{i}^{s \rightarrow d}}\left(t_{j}^{p}+\frac{F_{j}^{P}\left(t_{j}^{p}\right)}{f_{j}^{P}\left(t_{j}^{p}\right)}\right)\right] q_{i}\left(t^{p}\right)
$$


subject to constraints (13) and (14). Here, $P_{i}^{s \rightarrow d}$ denotes the set of nodes on the $i^{t h}$ path in $P^{s \rightarrow d}$. Also, the payment made to a node $j \in N$ is given by,

$$
\begin{array}{r}
p_{j}\left(t^{p}\right)=\frac{m_{j}}{c_{j}} \sum_{i \in P^{s} \stackrel{j}{\rightarrow} d} q_{i}\left(t^{p}\right)+\int_{m_{j} / c_{j}}^{b_{j} / v_{j}} \sum_{i \in P^{s^{j} \rightarrow d}} q_{i}\left(\psi_{j}, t_{-j}^{p}\right) d \psi_{j} \\
\forall j \in N
\end{array}
$$

Proof: Please refer [2] for the proof.

\section{A. Computing the route and payments}

In this paper, we focus on investigating the deployment the path auction scheme in a network in which each node $j \in N$ has sufficient capacity to route the sender's traffic. In other words, we consider $c_{j} \geq R, \forall j \in N$, so that the traffic from $s$ to $d$ can always be routed over a single path. However, note that, the theory of the path auction developed is generic and holds for the multi-path routing scenario as well.

Let us first define the following function,

$$
\zeta_{j}\left(t_{j}^{p}\right)=t_{j}^{p}+\frac{F_{j}^{P}\left(t_{j}^{p}\right)}{f_{j}^{P}\left(t_{j}^{p}\right)}, \quad \forall j \in N
$$

For any given $t \in T$, notice that $\zeta_{j}\left(t_{j}^{p}\right)$ is a constant for each $j \in N$. We refer to $\zeta_{j}\left(t_{j}^{p}\right)$ as the competence factor of node $j$. Also, the competence factor of path $i \in P^{s \rightarrow d}$ can be defined as $\sum_{j \in P_{i}^{s \rightarrow d}} \zeta_{j}\left(t_{j}^{p}\right)$. Based on the above definitions and lemma (3), $q$ should be such that the following function is minimized,

$$
\Phi\left(t^{p}\right)=\sum_{i=1}^{K}\left[\sum_{j \in P_{i}^{s \rightarrow d}} \zeta_{j}\left(t_{j}^{p}\right)\right] q_{i}\left(t^{p}\right)
$$

for all $t^{p}$ in $T^{p}$, subject to constraint (13).

The function $q(\cdot)$ : Clearly, (23) would get minimized for any $\bar{t}^{p} \in T^{p}$ while satisfying constraint (13) if $q$ assigns all traffic to the path in $P^{s \rightarrow d}$ having the least competence factor, which we consider as the most competent path. In essence, the path with the least competence factor is a path that minimizes the expected payment that the end-user has to make to have a packet routed from $s$ to $d$. We refer to such a path as the Expected Least Paid Route or simply ELPR. Formally, $q$ should assign all traffic to path $k$ in $P^{s \rightarrow d}$, such that,

$$
\sum_{j \in P_{k}^{s \rightarrow d}} \zeta_{j}\left(t_{j}^{p}\right)=\min _{1 \leq i \leq K} \sum_{j \in P_{i}^{s \rightarrow d}} \zeta_{j}\left(t_{j}^{p}\right)
$$

One can find the path to which all traffic should be assigned by using the competence factor $\zeta_{j}\left(t_{j}^{p}\right)$ as the weight of node $j \in N$ in the graph $G$ and using Dijkstra's algorithm to find the path from $s$ to $d$ having the minimum competence factor, which is the shortest path w.r.t node weights.

The function $p(\cdot)$ : As suggested by the payment formula (21), a node that does not route any traffic, i.e., is not included in the most competent path, does not receive any payment. This is because if $t_{j}=\left\{m_{j}, c_{j}\right\}$ is the type of node $j$ not on the most competent path, then we have $\sum_{i \in P^{s}{ }_{j}{ }_{d}} q_{i}\left(\psi_{j}, t_{-j}^{p}\right)=0, \forall \psi_{j} \in\left[\frac{m_{j}}{c_{j}}, \frac{b_{j}}{v_{j}}\right]$.

On the other hand, (21) dictates that node $j$ on the most competent path has to be paid an amount equal to the cost that $j$ would have incurred for forwarding a packet from $s$ if the type of $j$ had been associated with the highest possible per packet cost that allowed $j$ to be included on the most competent path. Specifically, let $t_{j}$ be the type of node $j$ on the most competent path. Also, let the difference between the competence factor of the most competent path in the graph $G$ and that of graph $G \backslash j$ be $\epsilon$. To be included on the most competent path in the graph $G, j$ 's type could have corresponded to all those types in $T_{j}$ for which its competence factor did not become more than or equal to $\zeta_{j}\left(t_{j}^{p}\right)+\epsilon$. Clearly, the highest possible per packet price that can be associated with these types is $\zeta^{-1}\left(\zeta_{j}\left(t_{j}^{p}\right)+\epsilon\right)$. Thus, $j$ has to be paid the minimum of $\zeta^{-1}\left(\zeta_{j}\left(t_{j}^{p}\right)+\epsilon\right)$ and $\frac{b_{j}}{v_{j}}$.

Based on the above argument, algorithm (1) presents the procedure for computing the most competent path (ELPR) in $G$ on which all traffic should be routed and the payment to be made to each node in $N$.

Algorithm 1 Single Path Routing- Computing Optimal Path and Payments

Require: $G=(V, E)$; source $s$; destination $d ; t=$ $\left(t_{1}, \cdots, t_{n}\right)$

Ensure: Most competent path $P$ (ELPR); Pay $p_{j}, \forall j \in N$

1: for all $j \in N$ do

2: $\quad t_{j}^{p} \leftarrow \frac{m_{j}}{c_{j}}$

3: $\quad \zeta_{j}\left(t_{j}^{p}\right) \leftarrow t_{j}^{p}+\frac{F_{j}^{P}\left(t_{j}^{p}\right)}{f_{j}^{P}\left(t_{j}^{p}\right)}$

4: end for

5: Using $\zeta_{j}\left(t_{j}^{p}\right)$ as the weight of node $j \in N$, find a path in $G$ from $s$ to $d$ that has the least competence factor. Denote the set of nodes in the selected path as $P$.

6: $p_{j} \leftarrow 0, \quad \forall j \in\{N \backslash P\}$.

7: for all $j \in P$ do

8: $\quad$ Using $\zeta_{k}\left(t_{k}^{p}\right)$ as the weight of node $k \in N \backslash j$, find a path in $G \backslash j$ from $s$ to $d$ with the least competence factor. Denote the set of nodes in the selected path as $S$.

9: $\quad \epsilon \leftarrow \sum_{j \in S} \zeta_{j}\left(t_{j}^{p}\right)-\sum_{j \in P} \zeta_{j}\left(t_{j}^{p}\right)$

10: $\quad p_{j} \leftarrow \min \left(\zeta^{-1}\left(\zeta_{j}\left(t_{j}^{p}\right)+\epsilon\right), \frac{b_{j}}{v_{j}}\right)$

11: end for

Algorithm (1) requires finding the shortest path from $s$ to $d$ w.r.t node weights. This can be done using Dijkstra's algorithm in $O(|E|+|V| \log |V|)$ time. Clearly, the overall running time of the algorithm is $O\left(|E||V|+|V|^{2} \log |V|\right)$.

$B$. Who pays the nodes?

If the sender pays the intermediate nodes, then it is possible that the intermediate nodes drop the packets instead of forwarding them, while collecting a payment for the same. To avoid this, we make the destination node make the payment to each intermediate node for every packet it receives. It is easy to see that, in such a scenario, no node will have an unilateral incentive to drop any fraction of traffic.

\section{Simulation Results}

For the purpose of simulations, nodes (in $N$ ) have been randomly placed in a $1000 \times 1000 \mathrm{~m}^{2}$ area, with the source $s$ and destination $d$ being placed near two opposite corners of the square. In the simulations we assume that $m_{j}$ and $c_{j}$ are independent random variables, i.e., $f_{j}^{M C}=f_{j}^{M} \cdot f_{j}^{C}$. For each node $j \in N$, its cost $m_{j}$ has been assumed to be uniformly 

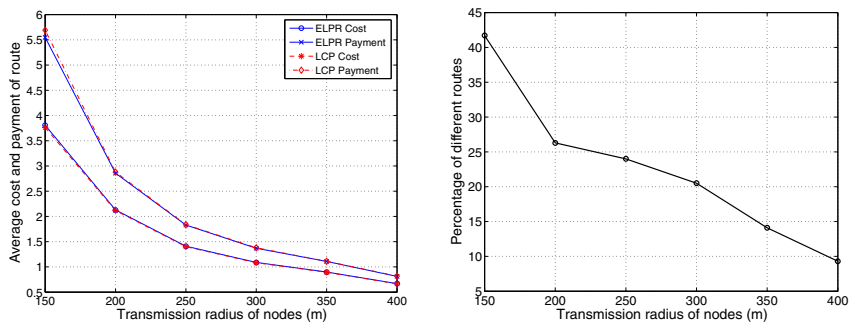

(a) Variation of cost and payment with (b) Percentage of routes for which transmission radius of the nodes.

ELPR and LCP are different.

Fig. 1. Single Path Routing.

distributed over $[1,4]$ and its capacity $c_{j}$ has been assumed to be uniformly distributed over $[2,7]$. All results have been averaged over 1000 independent runs. We assume that $s$ inputs data at $2 \mathrm{pkts} / \mathrm{sec}$ for all the experiments.

We will compare the performance of our algorithm with LCP routing. Recall that, in LCP routing, the type of a node corresponds to the per packet cost it owes to the primary spectrum holder for transmitting a packet over its spectrum.

\section{A. Varying Transmission Radius}

For Figures 1(a), 1(b) and 1(c) the number of nodes have been taken to be 200. Figure 1(a) shows how the cost of ELPR and LCP route and their corresponding payments decrease with increasing transmission radius of the nodes. The cost of a route refers to the summation of the costs incurred by the nodes on the route per packet routed by the network. The payment of a route is the summation of the payments received by the nodes on the route per packet delivered to $d$. As the transmission radius of the nodes increases, the path length (hop count) tends to decrease, thereby resulting in paths of lower cost to which lesser payments have to be made. Note that the cost of ELPR is only slightly larger than that of LCP. This implies that, as expected, ELPR is a route of relatively low cost, but not necessarily the route with the lowest cost. Further, the payment needed for ELPR is slightly less than that needed for LCP. This shows that ELPR can enforce truthfulness on both cost and capacity reporting of a node while requiring a payment that is less than that required by LCP, which only ensures truthful cost reporting. Note that the saving in payment by ELPR against LCP is more pronounced at lower transmission radius. This is because the total savings over the intermediate hops become more and more significant as the number of hops increase.

Figure 1(b) shows the percentage of cases (in the 1000 independent runs) in which ELPR and LCP were different routes, with varying transmission radius of the nodes. Clearly, the percentage of cases in which ELPR and LCP were different routes decrease with increasing transmission radius of the nodes. Thus, as the transmission radius increases, or in other words as the path length decreases, the chance of the path with the minimum cost being also the path that minimize the expected payment to be made per packet increases.

Figure 1(c) plots how the cost, weight (competence factor) and payment of ELPR increase with average number of hops.

\section{$B$. Varying number of nodes}

Figure 1(d) shows how the cost of ELPR and LCP route and their corresponding payments decrease as the total number of nodes is increased. The transmission radius of the nodes was set at $250 \mathrm{~m}$. As the number of nodes is increased, the number
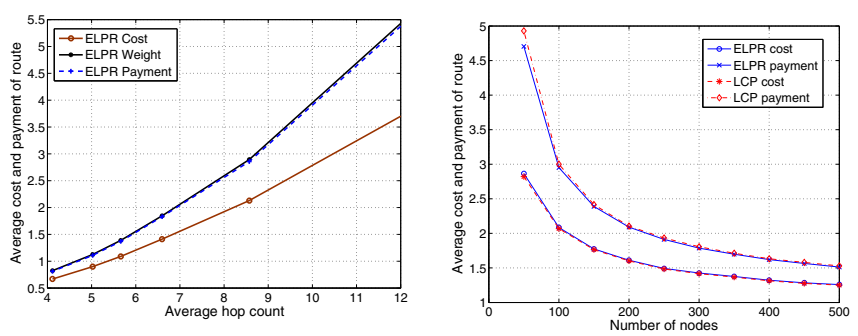

(c) Variation of cost, weight and pay- (d) Variation of cost and payment with ment of ELPR with avg. path length. number of nodes in the network.

of alternative paths between sender and destination increases and thus both ELPR and LCP starts corresponding to lower and lower cost routes. Accordingly, the payment needed for the routes also follows a decreasing trend. Note that the saving in payment by ELPR is more significant when the number of nodes is less. This is because of fewer alternative paths at lower number of nodes. When the number of alternative paths is less, the difference of cost (competence factor) between the best and the second best path for LCP (ELPR) tends to be higher than when the number of alternative paths is more. Thus, for fewer alternative paths, LCP requires a more higher payment than ELPR since VCG does not have an upper limit for payment to a node.

\section{CONCLUSIONS}

The problem of routing in DSA networks, where each secondary node has a privately known cost and capacity, is complicated by the fact that each node can potentially improve its utility by dishonestly reporting its type to the routing mechanism. We design a path auction based routing scheme for DSA networks that can enforce honest revealing of cost and capacity to be a dominant strategy for every node in the path auction game. Furthermore, our scheme also minimizes the payment that needs to be given to the nodes, thus making our scheme attractive to the end-user. We present a polynomial time algorithm for computing the optimal route over which to route traffic and the payment to be received by each node. Simulation results suggest that our path auction mechanism can ensure truthfulness on both cost and capacity while making a payment less than that required for VCG based LCP routing.

\section{REFERENCES}

[1] R. J. Berger, "Open spectrum: a path to ubiquitous connectivity", ACM Queue 1, 3, May 2003.

[2] S. Brahma and M. Chatterjee, "A Bayesian based Routing Mechanism for DSA Networks", Technical Report, University of Central Florida, January 2011.

[3] E. Clarke, "Multipart pricing of public goods", Public Choice 11, pp. 17-33, 1971

[4] J. Feigenbaum, C. Papadimitriou, R. Sami, and S. Shenker. "A BGP-based mechanism for lowest-cost routing", Distributed Computing, 18, pp. 61-72, 2005

[5] T. Groves. "Incentives in teams", Econometrica 41, pp. 617-663, 1973.

[6] J. Hershberger and S. Suri. "Vickrey prices and shortest paths: what is an edge worth?", IEEE Symp. on Foundations of Computer Science, pp. 252-259, 2001.

[7] J. Jia, Q. Zhang, Q. Zhang, and M. Liu. "Revenue generation for truthful spectrum auction in dynamic spectrum access", ACM MobiHoc, 2009, pp. 3-12.

[8] X. Y. Li, Y. Wu, P. Xu, G. Chen, and M. Li. "Hidden information and actions in multi- hop wireless ad hoc networks", ACM MobiHoc, pp. 283-292, 2008.

[9] R. B. Myerson. "Optimal auction design", Mathematics of Operations Research, 6(1), pp. 58-73, Feb 1981.

[10] N. Nisan and A. Ronen. "Algorithmic mechanism design", Games and Economic Behavior 35, pp. 166-196, 2001.

[11] T. Shu and M. Krunz. "Truthful Least-Priced-Path Routing in Opportunistic Spectrum Access Networks", IEEE Infocom, pp. 1037-1045, 2010.

[12] W. Vickrey. "Counterspeculation, auctions, and competitive sealed tenders", Journal of Finance 16, pp. 837, 1961.

[13] X. Zhou, S. Gandhi, S. Suri, and H. Zheng. "eBay in the sky: strategy-proof wireless spectrum auctions", ACM MobiCom, pp. 2-13, 2008.

[14] X. Zhou and H. Zheng, "TRUST: A General Framework for Truthful Double Spectrum Auctions", IEEE INFOCOM, pp. 999-1007, 2009. 